\title{
Exploring eukaryotic formate metabolisms to enhance microbial growth and lipid accumulation
}

\author{
Zhiguo Liu', Tolutola Oyetunde², Whitney D. Hollinshead², Anna Hermanns ${ }^{1,3}$, Yinjie J. Tang ${ }^{2}$, Wei Liao ${ }^{1}$ \\ and Yan Liư
}

\begin{abstract}
Background: C1 substrates (such as formate and methanol) are promising feedstock for biochemical/biofuel production. Numerous studies have been focusing on engineering heterologous pathways to incorporate $\mathrm{C} 1$ substrates into biomass, while the engineered microbial hosts often demonstrate inferior fermentation performance due to substrate toxicity, metabolic burdens from engineered pathways, and poor enzyme activities. Alternatively, exploring native C1 pathways in non-model microbes could be a better solution to address these challenges.
\end{abstract}

Results: An oleaginous fungus, Umbelopsis isabellina, demonstrates an excellent capability of metabolizing formate to promote growth and lipid accumulation. By co-feeding formate with glucose at a mole ratio of 3.9:1, biomass and lipid productivities of the culture in $7.5 \mathrm{~L}$ bioreactors were improved by 20 and $70 \%$, respectively. ${ }^{13} \mathrm{C}$-metabolite analysis, genome annotations, and enzyme assay further discovered that formate not only provides an auxiliary energy source [promoting NAD(P)H and ATP] for cell anabolism, but also contributes carbon backbones via folatemediated C1 pathways. More interestingly, formate addition can tune fatty acid profile and increase the portion of medium-chain fatty acids, which would benefit conversion of fungal lipids for high-quality biofuel production. Flux balance analysis further indicates that formate co-utilization can power microbial metabolism to improve biosynthesis, particularly on glucose-limited cultures.

Conclusion: This study demonstrates Umbelopsis isabellina's strong capability for co-utilizing formate to produce biomass and enhance fatty acid production. It is a promising non-model platform that can be potentially integrated with photochemical/electrochemical processes to efficiently convert carbon dioxide into biofuels and value-added chemicals.

Keywords: ${ }^{13} \mathrm{C}, \mathrm{NAD}(\mathrm{P}) \mathrm{H}$, Fatty acids, Flux balance analysis, One-carbon metabolic pathway

\section{Background}

Microbial one-carbon (C1) metabolism plays a critical role in global carbon cycles [1]. Key C1 molecules include carbon dioxide, methane, carbon monoxide, and methanol. Among these $\mathrm{C} 1$ compounds, $\mathrm{CO}_{2}$ is one of the most abundant greenhouse gases in the Earth's atmosphere, which contributes up to $26 \%$ of the global greenhouse effect [2]. Developing technologies to fix and utilize

\footnotetext{
*Correspondence: liuyan6@msu.edu

1 Department of Biosystems and Agricultural Engineering, Michigan State University, East Lansing, MI 48824-1323, USA

Full list of author information is available at the end of the article
}

$\mathrm{CO}_{2}$ has attracted increasing attention to alleviate the impact of greenhouse gas emissions on climate change. Numerous studies have been conducted on microbial C1 metabolism, such as autotrophic $\mathrm{CO}_{2}$ fixation, and methylotrophic or methanotrophic carbon utilization (from $\mathrm{CO}_{2}$ reduction reaction) [3-5]. In general, autotrophic $\mathrm{CO}_{2}$ fixation faces challenges including mass transfer limitation, low metabolic rate of adenosine triphosphate (ATP)/reduced nicotinamide adenine dinucleotide $[\mathrm{NAD}(\mathrm{P}) \mathrm{H}]$ generations, and poor enzymatic synthesis activities. Alternatively, formate, a stable $\mathrm{C} 1$ compound [6], can be generated from $\mathrm{CO}_{2}$ using electrochemical 
reduction under mild reaction conditions and proper catalysts [6,7]. Due to its solubility and reducing power, formate is a promising feedstock to support microbial growth.

Bacterial formate utilization has been intensively studied in methylotrophs and lithoautotrophs [7-10]. There are two interdependent processes naturally existed to achieve formate metabolism: formate oxidation and formate assimilation [11-13]. The formate oxidation relies on $\mathrm{NAD}(\mathrm{P}) \mathrm{H}$-dependent formate dehydrogenase (FDH) that transfers electrons from formate to $\mathrm{NAD}(\mathrm{P}) \mathrm{H}$, and facilitates ATP synthesis to support cell growth [14-16]. For example, Ralstonia eutropha uses the formate oxidation to gain ATP and $\mathrm{NAD}(\mathrm{P}) \mathrm{H}$, and to support the Calvin Cycle to fix $\mathrm{CO}_{2}$ [1]. In contrast, the formate assimilation directly incorporates formate via five possible pathways including the serine pathway, the reductive acetyl-CoA pathway, the ribulose monophosphate pathway, the xylulose 5 -phosphate pathway, and the reductive glycine pathway $[1,17]$. All of these pathways start from the reduction of formate to methylenetetrahydrofolate (methylene-THF). For example, some methylotrophs first convert formate into formyl-THF that is reduced to methylene-THF. The methylene-THF then donates its formaldehyde for serine generation, and leads to production of acetyl-CoA [1]. According to these theoretical analyses, metabolic engineers have been devoting tremendous efforts to develop model bacterial platform for C1 utilization [18-20]. Corynebacterium glutamicum and E. coli were two of the model strains that have been engineered to co-utilize $\mathrm{C} 1$ substrates, but bacterial platform shows deleterious growth and poor carbon utilizations.

As for eukaryotes, a few species can tolerate and utilize formate, and most of them use formate oxidation pathway to release $\mathrm{NAD}(\mathrm{P}) \mathrm{H}$ from formate to support their growth [21-25]. For instance, with the addition of formate, Penicillin G production of Penicillium chrysogenum fermentation was significantly improved [26]. In this study, we focus on an oleaginous fungus, Umbelopsis isabellina, that has demonstrated the capability to co-utilize formate and other carbon sources for growth and lipid accumulation. To explore fungal formate assimilation pathways and co-metabolism on diverse carbon substrates, this study has (1) used ${ }^{13} \mathrm{C}$ tracing to elucidate formate utilization pathways in $U$. isabellina, (2) applied biochemistry assay to delineate the effects of formate on fungal growth and fatty acid accumulation, and (3) rigorously quantified C1 carbon contributions to biomass synthesis.

\section{Methods}

Strain, seed culture, and reagents

Umbelopsis Isabellina ATCC 42613 was obtained from the American Type Culture Collection (Manassas, VA). Spores were washed by sterile deionized water and collected after cultivation on potato dextrose agar (PDA) medium at $30{ }^{\circ} \mathrm{C}$ for two weeks. Seed was cultured in $24 \mathrm{~g} \mathrm{~L}^{-1}$ potato dextrose broth (PDB) medium with $8 \mathrm{~g} \mathrm{~L}^{-1}$ yeast extract (YE) (Neogen, Lansing), at room temperature of $25{ }^{\circ} \mathrm{C}$ and a shaking speed of $180 \mathrm{rpm}$ for $36 \mathrm{~h}$. Inoculation size was $5 \%(\mathrm{v} / \mathrm{v})$ if not specified. Chemicals, substrates (except ${ }^{13} \mathrm{C}$-formate from Cambridge Isotope Laboratories, Inc., Tewksbury, MA), and reagents were purchased from Sigma-Aldrich (SigmaAldrich, St. Louis, MO).

\section{Fermentation}

Batch fermentation was conducted using shaking flasks and fermenters. For the shaking flask culture, 2-L Erlenmeyer flasks containing $0.7 \mathrm{~L}$ medium were placed on a New Brunswick Shaker at $180 \mathrm{rpm}$ and cultured at $25^{\circ} \mathrm{C}$. For the fermenter culture, 7.5-L fermenters (New Brunswick-Eppendorf, Bioflo 115, Hauppauge, NY) were used to carry out the experiment. The fermenters contained 3 $\mathrm{L}$ medium. The airflow rate was $1 \mathrm{vvm}$, and the agitation speed was $200 \mathrm{rpm}$. The flask culture of formate medium contained $0.5 \mathrm{~g} \mathrm{~L}^{-1} \mathrm{YE}, 2 \mathrm{~g} \mathrm{~L}^{-1}{ }^{13} \mathrm{C}$-labeled formate, and trace elements [27]. The flask culture of glucose and formate medium contained $0.5 \mathrm{~g} \mathrm{~L}^{-1} \mathrm{YE}, 4 \mathrm{~g} \mathrm{~L}^{-1}{ }^{13} \mathrm{C}$-labeled formate (99\% purity), $10 \mathrm{~g} \mathrm{~L}^{-1}$ glucose, and trace elements. The duration of all flask cultures was $72 \mathrm{~h}$. The medium for the fermenter culture contained $1 \mathrm{~g} \mathrm{~L}^{-1} \mathrm{YE}$, $10 \mathrm{~g} \mathrm{~L}^{-1}$ glucose and $4 \mathrm{~g} \mathrm{~L}^{-1}{ }^{13} \mathrm{C}$-labeled formate (the control experiment excluded formate supplement). The duration of fermenter culture was $48 \mathrm{~h}$. The $\mathrm{pH}$ of the flask cultivation was maintained at 6.0 by manually adding $1 \mathrm{M}$ sulfuric acid or sodium hydroxide every $6 \mathrm{~h}$ in first $12 \mathrm{~h}$ and every $12 \mathrm{~h}$ afterwards. The $\mathrm{pH}$ of the fermenter cultivation was automatically maintained at 6.0 using $1 \mathrm{M}$ sulfuric acid or sodium hydroxide. Samples were taken periodically for biomass composition and metabolite measurement.

\section{Metabolite and biomass composition analyses}

Formate and glucose in the fermentation broth were determined by a High-Performance Liquid Chromatography (HPLC) (Shimadzu Prominence, Japan) equipped with a Bio-rad Aminex HPX-87H analytical column and 
a refractive index detector [27]. As for NADH measurement, one $\mathrm{ml}$ broth was centrifuged at $4000 \mathrm{rpm}$ for $1 \mathrm{~min}$ and the precipitated biomass was washed by cold phosphate buffered saline (PBS) before the NADH was measured by the NAD+/NADH Quantification Colorimetric Kit (BioVision, San Francisco). Standard solutions were prepared and measured on daily basis.

Fungal biomass with the known volume of fermentation broth was collected by vacuum filtration and washed three times by deionized water before drying at $105{ }^{\circ} \mathrm{C}$ overnight for dry matter measurement. Lipid extraction of the fungal biomass was conducted using the Bligh and Dyer method [28], and the lipid content was determined gravimetrically. The extracted lipid was then subjected to methanolysis to turn fatty acids into methyl esters [29]. The resulting methyl esters were further analyzed by gas chromatography-mass spectrometry (GC-MS) to obtain the fatty acid profile [27]. F.A.M.E. Mix (C4-C24) with serial dilutions $(100,50,25,12.5,6.25,3.125,1.5625$, $0.78125 \mu \mathrm{g} \mathrm{mL}^{-1}$ ) and chloroform was applied as external standard. Samples were diluted ten times prior to analysis to reach reasonable detection levels. All samples and standards were spiked with $50 \mu \mathrm{g} \mathrm{mL} \mathrm{m}^{-1}$ methyl nonadecanoate (C19:0) as internal standard.

\section{Carbon isotopomer analysis}

Biomass samples were harvested by centrifuge, and the cell pellets were hydrolyzed with $6 \mathrm{~mol} \mathrm{~L}^{-1} \mathrm{HCl}$ at $100^{\circ} \mathrm{C}$ overnight. The resulting amino acid mixtures were subsequently air dried and derivatized with $N$-tert-butyldimethylsilyl- $N$-methyltrifluoroacetamide (TBDMS) prior to GC-MS analysis. A published software was used to analyze and correct amino acid MS data, as described in the previous report [30]. The fragments [M-57] or [M-15], containing the entire carbon backbone of the amino acids, were used to demonstrate the incorporation of ${ }^{13} \mathrm{C}$-formate. Results are described using $m+0, m+1$, and $m+2$ for unlabeled, singly labeled, doubly labeled amino acids, and so on.

Flux balance analysis (FBA) of fungal formate co-utilization To understand the influence of fungal formate metabolism on biomass growth, a genome-scale yeast model, iMM904 (bigg.ucsd.edu), was adapted to provide stoichiometric insights into formate-glucose co-metabolism [31]. The model was modified by adding a pathway from formate to methylene-THF. The growth rates were predicted based on different glucose/formate co-utilization influxes and the objective function of maximal biomass production. The FBA simulation using COBRA tool box can then determine the carbon flows from formate to biomass synthesis (formate assimilation) and to $\mathrm{CO}_{2}$ release for $\mathrm{NAD}(\mathrm{P}) \mathrm{H}$ generations (formate oxidation).

\section{Results and discussion}

Formate as an energy and carbon source to enhance the growth of $U$. isabellina

The isotopomer analysis [32] provided the labeling information of critical precursor metabolites during formate assimilation such as pyruvate, acetyl-CoA, methyleneTHF, formyl-THF, glycine, and serine (Fig. 1). The fungal culture on ${ }^{13} \mathrm{C}$-formate supplemented with yeast extract shows that eight amino acids (alanine, aspartate, glutamate, glycine, leucine, methionine, phenylalanine, and serine) were detected with significant labeling extent (Fig. 2a). Among them, methionine and serine are the amino acids with a large amount of $m+2$ (e.g., over $10 \%$ of methionine was labeled with two carbons). Besides, methionine labeling enrichment is significantly $(\mathrm{P}<0.05)$ higher than its precursor aspartate, indicating the labeling of Methyl-THF unit (Asp + MethylTHF $\rightarrow$ methionine). This result demonstrates that $U$. isabellina assimilates ${ }^{13} \mathrm{C}$-formate via folate-mediated $\mathrm{C} 1$ pathways to generate Methyl-THF (Fig. 1). On the other hand, the TCA cycle-derived amino acids (aspartate and glutamate) show enriched ${ }^{13} \mathrm{C}(15 \sim 30 \%$, mostly $m+1)$, which indicates that the strain also uses anaplerotic pathway (PEP $+\mathrm{CO}_{2} \rightarrow$ Oxaloacetate) to fix the $\mathrm{CO}_{2}$ from formate oxidation (Fig. 1). Similar labeling distributions were observed when adding formate in glucose medium (Fig. 2b). The ${ }^{13} \mathrm{C}$-fingerprinting provided the evidence of formate contribution to fungal growth through both formate assimilation and oxidation pathways. However, the experimental data also demonstrate that formate is not an efficient carbon source to support high-yield biomass growth (Table 1). The biomass accumulation on the formate medium was much lower than the culture on the glucose medium. Mixing formate with other carbon sources could be a good solution to synergistically utilize carbon and energy contents in formate and enhance carbon utilization efficiency of the fermentation. Therefore, the effects of formate as a supplemental carbon source on fungal metabolism was further studied.

Umbelopsis isabellina growth on glucose media with/ without ${ }^{13} \mathrm{C}$-formate demonstrates that formate as a supplemental carbon and energy source significantly $(\mathrm{P}<0.05)$ improved the fungal biomass accumulation (Fig. 3a). At the end of the culture (48 h), the cultivations with and without formate reached 4.3 and 
a

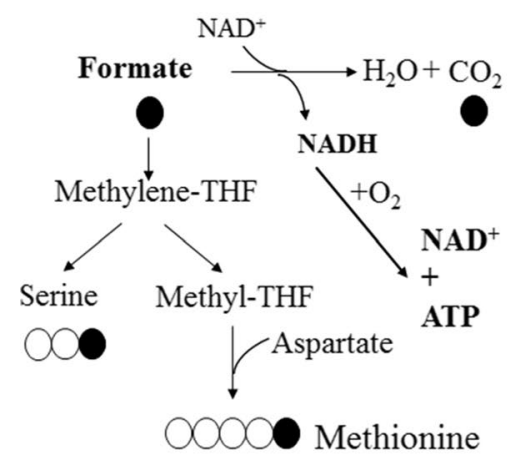

C1 metabolism

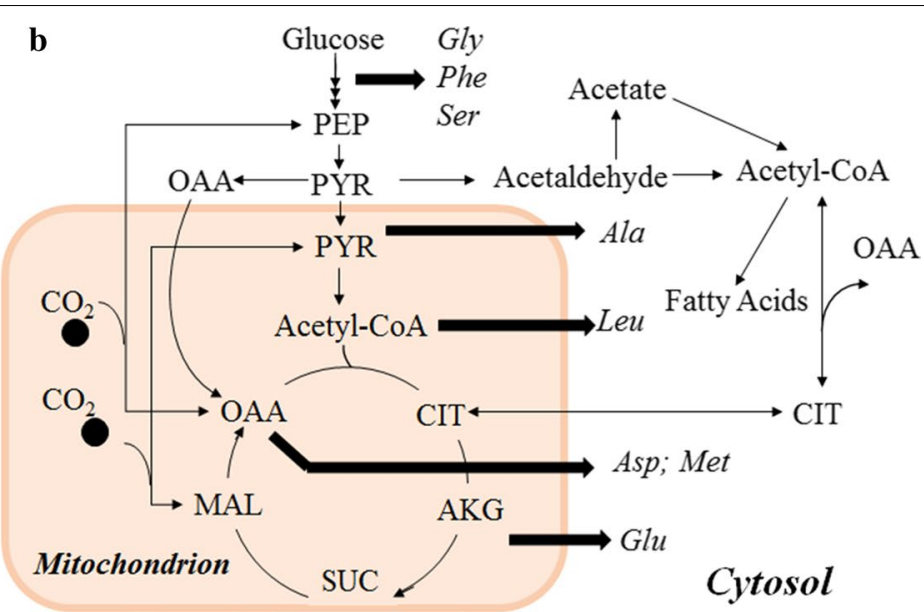

Fig. 1 Simplified pathways of carbon and energy metabolism during U. isabellina culture on glucose and formate. a Formate metabolism. b Glucose metabolism

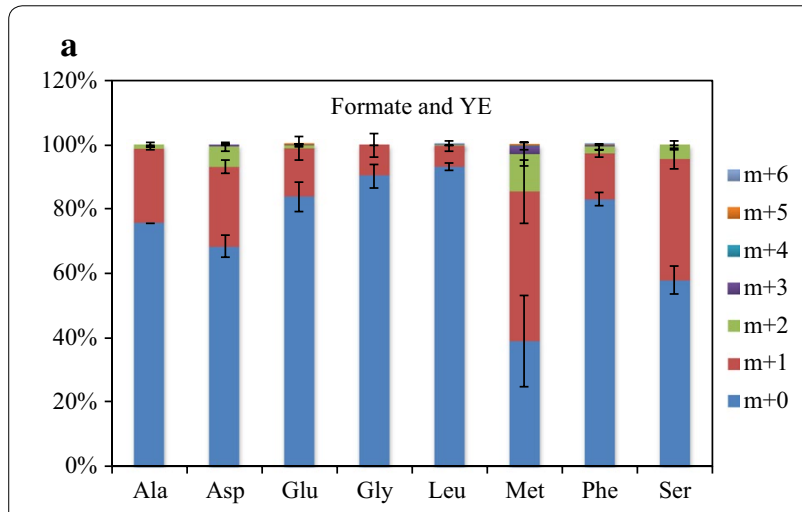

Table $1 U$. isabellina growth on the media with formate and glucose as individual carbon sources

\begin{tabular}{llll}
\hline & \multicolumn{3}{l}{ Biomass concentration $\left(\mathbf{g ~ L}^{-\mathbf{1}}\right)$} \\
\hline Culture time $(\mathrm{h})$ & 0 & 48 & 96 \\
Formate medium & $0.31 \pm 0.03$ & $0.58 \pm 0.04$ & $0.58 \pm 0.08$ \\
Glucose medium & $0.21 \pm 0.02$ & $3.67 \pm 0.43$ & $7.58 \pm 0.28$ \\
\hline
\end{tabular}

Data are average of three replicates with standard deviation

$3.8 \mathrm{~g} \mathrm{~L}^{-1}$ biomass, respectively. The data also demonstrate that the formate and glucose were simultaneously consumed without glucose catabolite repression. Formate also influences intracellular NADH concentrations (Fig. 3b). NADH in the culture with formate peaked at $352 \mathrm{pmol} \mathrm{mg}^{-1}$ dry biomass at $24 \mathrm{~h}$ (in the middle-log phase growth). In contrast, the control culture without formate had a much lower NADH at the same growth phase. When glucose and formate were depleted at the end of fermentations, NADH contents in both cultures were decreased to $\sim 110 \mathrm{pmol} \mathrm{mg}^{-1} \mathrm{dry}$ biomass. This phenomenon confirms the role of formate as an auxiliary energy source to alleviate glucose catabolic burdens for NADH production and save glycolysis metabolites for biosynthesis [26]. Since glucose shows no catabolite repression on formate metabolism, the use of formate to relieve energy burden could be a preferable technique to improve performance of microbial cultivation [33]. 

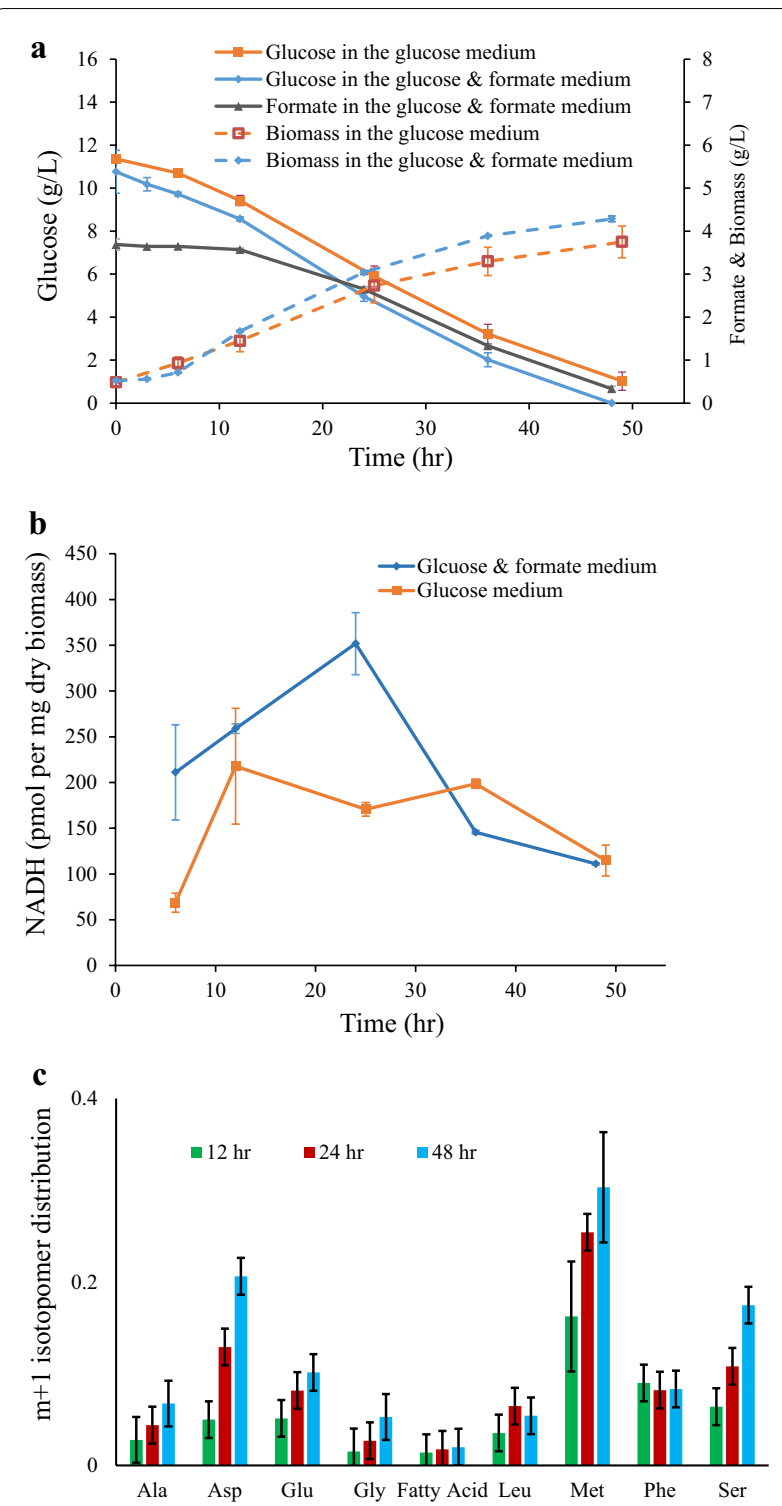

Fig. 3 Kinetics of fermentor culture using ${ }^{13} \mathrm{C}$-formate and glucose as the carbon sources. Data are average of two replicates with standard error. a Growth and substrate consumption kinetics; b NADH level kinetics; c Mass isotopomer $m+1$ fraction of proteinogenic amino acids for biomass collected at 12, 24, and 48 h. Fatty acid labeling estimated based on labeling of acetyl-CoA, which was determined from the first two carbons of leucine

During the culture on the glucose medium with ${ }^{13} \mathrm{C}$-formate, fungal proteinogenic amino acids demonstrate continuous ${ }^{13} \mathrm{C}$ enrichment, especially for serine and methionine (Fig. 3c). Fractions of labeled serine and methionine reached to 20 and $30 \%$, respectively, by the end of the culture. This result well aligns with the THFmediated formate assimilation pathway that is closely related with the serine cycle and methionine metabolism [1]. Both of them facilitate the transmethylation reactions [34]. In addition, the experimental data show that percentages of $m+1$ incorporated into aspartate and methionine (oxaloacetate-derived amino acids) were increased during the cultivation process (Fig. 3c). Aspartate labeling enrichment indicates that ${ }^{13} \mathrm{CO}_{2}$ released from formate oxidation was re-incorporated into the TCA cycle via the anaplerotic reactions [35]. A BLAST was then conducted to search for the related enzymes in formate pathways of $U$. isabellina (Table 2). Key enzymes were blasted using a stand-alone database built from genomic sequence of $U$. isabellina and the tBLASTn search engine. The BLAST results confirmed enzymes involving in formate oxidation and assimilation (all e-values $<10^{-40}$ ), demonstrating its native formate utilization capability.

\section{Effects of formate on lipid synthesis}

Lipid accumulation during the fermentation was also influenced by formate (Fig. 4). The lipid contents in the fungal biomass at the end of cultivation reached 43 and $38 \mathrm{~g}$ lipid/100 g dry fungal biomass for the media with and without formate, respectively. Compared to the control culture (glucose medium only), the culture on the glucose plus formate medium significantly $(\mathrm{P}<0.05)$ increased biomass yield (up to $13 \%$ ), biomass productivity (up to $20 \%$ ), lipid yield (up to $30 \%$ ), and lipid productivity (up to $70 \%$ ) (Fig. 4). As discussed in the previous section, formate co-metabolism alleviates the glucose dissimilation burden, and subsequently allows more glucose carbon to flow into lipid.

Interestingly, adding formate also changed the fatty acids profile (Fig. 5). The fatty acid biosynthesis includes recurring reactions that require a large amount of reducing factors and ATP [36]. The energy from formate oxidation may re-program fatty acid profiles. Short- and medium-chain fatty acids (C6:0 to $\mathrm{C} 16: 0)$ were all upregulated, while most of long-chain fatty acids (C17:0 to $\mathrm{C} 22: 1$ ) were downregulated with the addition of formate (Fig. 5). Medium-chain fatty acids are widely used in detergents and lubricants due to their lathering and low-viscosity properties [37]. However, yeast species usually did not produce short- and medium-chain fatty acids 
Table 2 Key enzymes relevant to formate pathways identified by blasting the $U$. isabellina genome

\begin{tabular}{|c|c|}
\hline Name & Reaction \\
\hline NAD-dependent formate dehydrogenase (FDH, EC 1.2.1.1) & Formate $+\mathrm{NAD}^{+} \leftrightarrow \mathrm{CO}_{2}+\mathrm{NADH}+\mathrm{H}^{+}$ \\
\hline Formate tetrahydrofolate ligase (EC6.3.4.3) & $\begin{array}{l}\text { ATP }+ \text { formate }+ \text { tetrahydrofolate } \leftrightarrow \text { ADP }+ \text { phosphate }+10 \text {-for- } \\
\text { myltetrahydrofolate }\end{array}$ \\
\hline $\begin{array}{l}\text { Methenyltetrahydrofolate cyclohydrolase/dehydrogenase (EC3.5.4.9 and } \\
\text { 1.5.1.5/15) }\end{array}$ & 5,10-Methenyltetrahydrofolate $+\mathrm{H}_{2} \mathrm{O} \leftrightarrow$ 10-formyltetrahydrofolate \\
\hline Glycine dehydrogenase (EC1.4.1.10) & Glycine $+\mathrm{H}_{2} \mathrm{O}+\mathrm{NAD}+\leftrightarrow$ glyoxylate $+\mathrm{NH}_{3}+\mathrm{NADH}+\mathrm{H}^{+}$ \\
\hline Aminomethyltransferase (EC2.1.2.10) & $\begin{array}{l}\text { Glycine }+ \text { tetrahydrofolate }+\mathrm{NAD}^{+} \leftrightarrow \text { 5,10-methylene-tetrahydro- } \\
\text { folate }+ \text { ammonium }+\mathrm{CO}_{2}+\mathrm{NADH}\end{array}$ \\
\hline Dihydrolipoyl dehydrogenase (EC1.8.1.4) & $\begin{array}{l}\text { Protein N6-(dihydrolipoyl)lysine }+\mathrm{NAD}^{+} \leftrightarrow \text { protein N6-(lipoyl) } \\
\text { lysine }+\mathrm{NADH}+\mathrm{H}^{+}\end{array}$ \\
\hline Serine hydroxymethyltransferase (EC2.1.2.1) & $\begin{array}{l}\text { 5,10-Methylenetetrahydrofolate }+ \text { glycine }+\mathrm{H}_{2} \mathrm{O} \leftrightarrow \text { tetrahydro- } \\
\text { folate }+ \text { L-serine }\end{array}$ \\
\hline Serine deaminase (EC4.3.1.17) & L-serine $\leftrightarrow$ pyruvate $+\mathrm{NH}_{3}$ \\
\hline
\end{tabular}

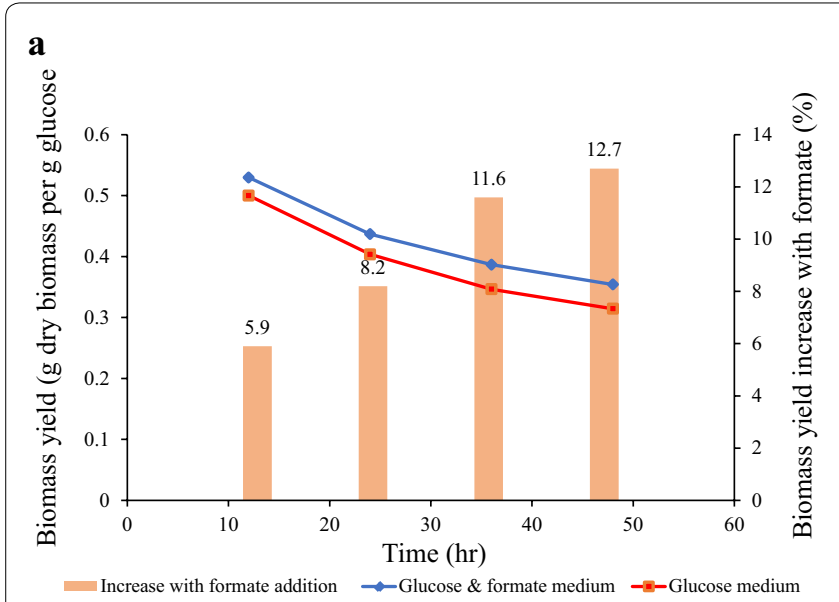

c

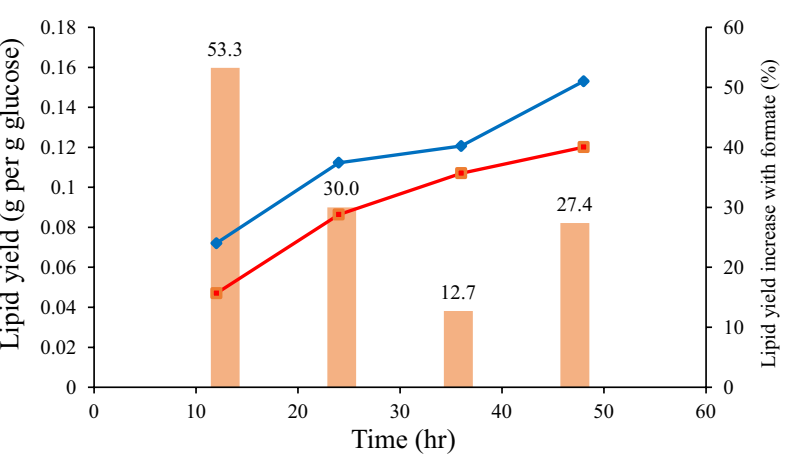

Increase with formate addition $\rightarrow$ Glucose \& formate medium $\quad-$ - Glucose medium

b
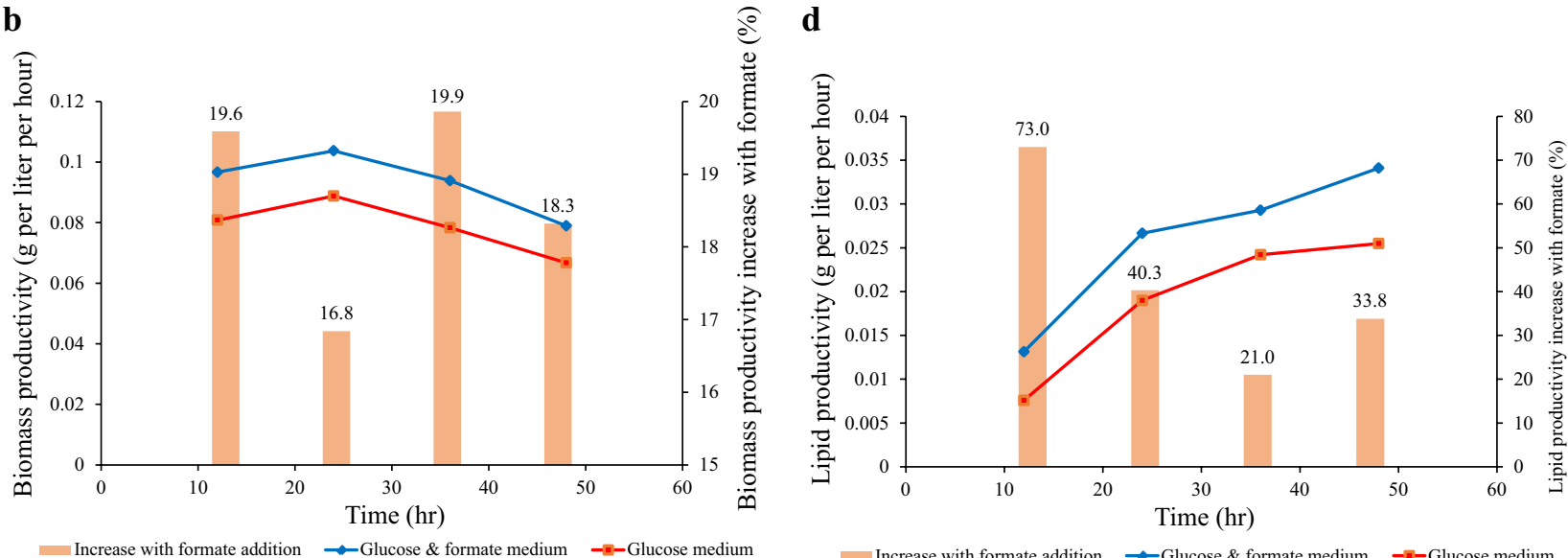

Fig. 4 Enhancement of biomass and lipid accumulation by formate supplement. Data are average of two replicates with standard error. a Biomass yield; $\mathbf{b}$ biomass productivity; $\mathbf{c}$ lipid yield; $\mathbf{d}$ lipid productivity 


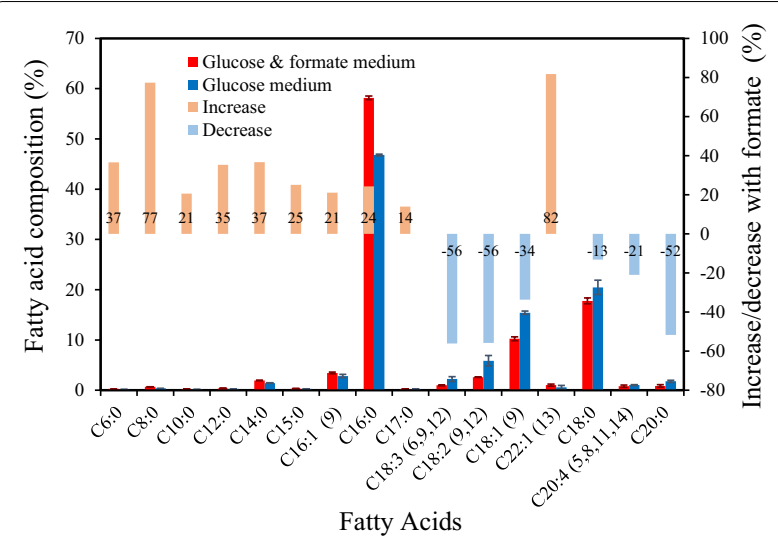

Fig. 5 Fatty acid composition profile shift with formate. Data are average of two replicates with standard error

except for engineered strains or cultures growing in synthetic media with high $\mathrm{C} / \mathrm{N}$ ratios and low culture temperature [37]. Therefore, using formate to control fatty acid profile can be applicable in industrial fermentation processes.

\section{Flux balance analysis (FBA) of fungal growth on glucose and formate}

Glucose/formate uptake rates and biomass accumulation from the experiments were used to carry out FBA (Table 3). The FBA results verify the positive effects of formate on fungal biomass accumulation and ATP generations (Fig. 6). First, formate oxidation significantly contributes to ATP generation through respirations (formate $\rightarrow \mathrm{NADH} \rightarrow \mathrm{ATP}$ ), particularly at lower glucose uptake rate (Fig. 6a). ATPs were released from mitochondria when cell metabolism aerobically consumes both glucose and formate (Fig. 6a). Since this process requires a large amount of dissolved oxygen, high aeration is needed to maintain the formate oxidation of ATP generation. Second, the amount of formate carbon used for the biomass accumulation (through folate metabolism) depends on both glucose and formate uptake rates. Lower glucose and higher formate uptake rates are beneficial for formate assimilation (Fig. 6b, c, d). For instance, at high glucose uptake rates of $0.2-0.8 \mathrm{mmol}$ glucose $\mathrm{g}^{-1}$ dry cell weight (DCW) $\mathrm{h}^{-1}$, formate was mainly used as the energy source and contributed little to biomass production (Fig. 6b). Once the glucose uptake rate dropped to $0.15 \mathrm{mmol}$ glucose $\mathrm{g}^{-1} \mathrm{DCW} \mathrm{h} \mathrm{h}^{-1}$, increasing formate utilization from $0 \mathrm{mmol}$ glucose $\mathrm{g}^{-1} \mathrm{DCW} \mathrm{h}{ }^{-1}$ to $5 \mathrm{mmol}$ glucose $\mathrm{g}^{-1}$ DCW $\mathrm{h}^{-1}$ promoted biomass production (Fig. 6d). It is apparent that formate becomes both energy and carbon sources under glucose-limiting conditions (Fig. 6c). Third, the FBA also demonstrates in silico growth with formate in the absence of glucose (Fig. 6d). Under a high formate uptake rate of $5 \mathrm{mmol}$ formate $\mathrm{g}^{-1} \mathrm{DCW} \mathrm{h}^{-1}$, the $\mathrm{C} 1$ assimilation pathways that $U$. isabellina possesses led to incorporation of $8 \%$ formate carbon into fungal biomass, while the remaining formate was oxidized as the energy source. The simulation results theoretically conclude the potential of formate as a carbon and energy source to support fungal growth. Although genetically modified organisms have been developed to use $\mathrm{C} 1$ feedstock, these engineered platform shows poor $\mathrm{C} 1$ utilizations (consumes less than $0.5 \mathrm{~g} \mathrm{~L}^{-1} \mathrm{C} 1$ substrate) and slow fermentation processes [18-20]. $U$. isabellina offers an alternative non-model platform, and future metabolic engineering efforts should focus on development of genetic tools to re-program its metabolisms to produce diverse products by co-utilization of formate with other organic substrates.

\section{Conclusions}

The results conclude that formate and glucose could be simultaneously utilized without catabolite repression. Formate was identified as a carbon and energy source in the culture of $U$. isabellina based on ${ }^{13} \mathrm{C}$-fingerprinting analysis and kinetic study. THF-based $\mathrm{C} 1$ assimilation pathways were counted for incorporation of formate into biomass. Extra NADH-dependent energy generated from formate oxidation led to enhanced biomass accumulation and elevated fatty acid synthesis as well as a significant

Table 3 Formate and glucose uptake rates and biomass accumulation

\begin{tabular}{|c|c|c|c|c|c|c|}
\hline \multirow[t]{2}{*}{ Fermentation medium } & \multicolumn{2}{|c|}{ Glucose uptake $\left(\mathrm{mmol} \mathrm{g}^{-1} \mathrm{DCW} \mathrm{h}^{-1}\right)$} & \multicolumn{2}{|c|}{ Formate uptake $\left(\mathrm{mmol} \mathrm{g}^{-1} \mathrm{DCW} \mathrm{h}^{-1}\right)$} & \multicolumn{2}{|c|}{$\begin{array}{l}\text { Biomass accumulation }\left(\mathrm{mmol} \mathrm{g}^{-1}\right. \\
\text { DCW } \mathrm{h}^{-1} \text { ) }\end{array}$} \\
\hline & $\begin{array}{l}\text { Glucose \& formate } \\
\text { medium }\end{array}$ & $\begin{array}{l}\text { Glucose } \\
\text { medium }\end{array}$ & $\begin{array}{l}\text { Glucose \& formate } \\
\text { medium }\end{array}$ & $\begin{array}{l}\text { Glucose } \\
\text { medium }\end{array}$ & $\begin{array}{l}\text { Glucose } \& \text { formate } \\
\text { medium }\end{array}$ & $\begin{array}{l}\text { Glucose } \\
\text { medium }\end{array}$ \\
\hline 3- L fermenter & 0.609 & 0.576 & 0.499 & - & 0.054 & 0.031 \\
\hline 3-L fermenter & 0.505 & 0.577 & 0.816 & - & 0.027 & 0.037 \\
\hline 0.7-L flask & 0.484 & 0.397 & 0.345 & - & 0.012 & 0.010 \\
\hline 0.7-L flask & 0.026 & - & 0.117 & - & 0.017 & - \\
\hline
\end{tabular}



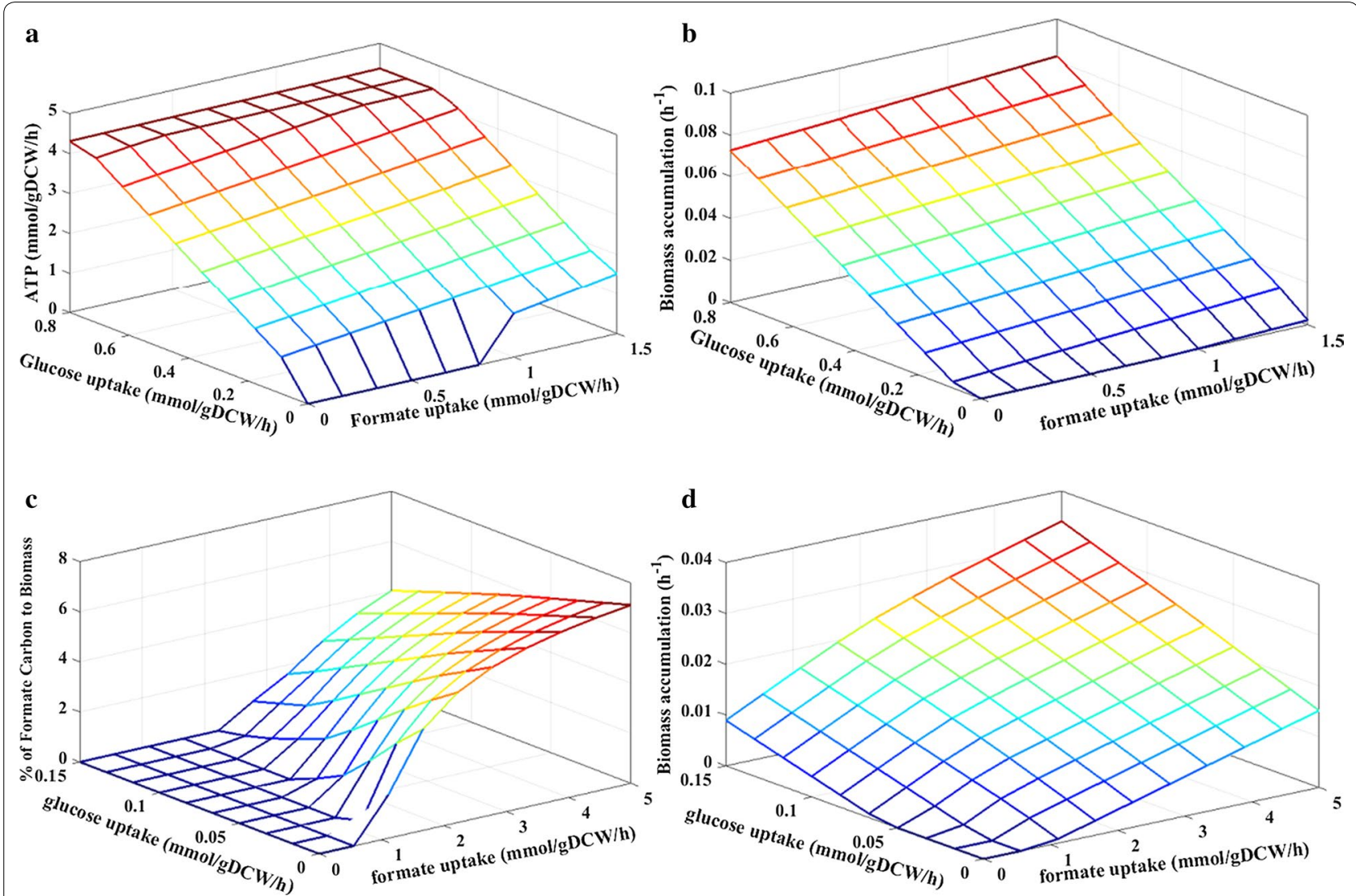

Fig. 6 Results of FBA analysis. Oxygen flux is limited to $2 \mathrm{mmol} \mathrm{g}^{-1} \mathrm{DCW} \mathrm{h}{ }^{-1}$ and maintenance flux is $1 \mathrm{mmol} \mathrm{ATP} \mathrm{g}^{-1} \mathrm{DCW} \mathrm{h}^{-1}$. a Effects of formate and glucose utilization on ATP production. $\mathbf{b}$ Effects of formate and glucose uptake rates on biomass growth. $\mathbf{c}$ Percentage of formate carbon to biomass. $\mathbf{d}$ Effects of formate and glucose uptake rates on biomass growth at low glucose utilization rates

shift in carbon chain length of the fatty acid profile. The FBA results further delineate the benefit of formate cometabolism for fungal biomass accumulation. This study clearly presents a new microbial chassis that $\mathrm{CO}_{2}$ utilization (via formate) and lignocellulosic sugar conversion can be synergistically coupled to produce biofuels and value-added chemicals, so that overall carbon utilization efficiency can be significantly enhanced. Future studies should focus on genetic tools to modify $U$. isabellina for efficient utilization of formate and improvement of fatty acid profile.

\section{Abbreviations}

$\mathrm{CO}_{2}$ : carbon dioxide; ATP: adenosine triphosphate; $\mathrm{NAD}(\mathrm{P}) \mathrm{H}$ : reduced nicotinamide adenine dinucleotide; NADH: nicotinamide adenine dinucleotide; FDH: formate dehydrogenase; Methylene-THF: methylenetetrahydrofolate; PDA: potato dextrose agar; PDB: potato dextrose broth; YE: yeast extract; HPLC high-performance liquid chromatography; PBS: phosphate buffered saline; GC-MS: gas chromatography-mass spectrometry; TBDMS: N-tert-butyldimethylsilyl-N-methyltrifluoroacetamide; FBA: flux balance analysis; DCW: dry cell weight.

\section{Authors' contributions}

$\mathrm{ZL}, \mathrm{AH}, \mathrm{YJT}, \mathrm{WDH}, \mathrm{YL}$ and $\mathrm{WL}$ conceived and designed the study. ZL, $A H$, and YL conducted experiments. ZL, TO, WDH, AH, YJT, WL, and YL analyzed and interpreted data, and wrote the manuscript. All authors read and approved the final manuscript.

\section{Author details}

${ }^{1}$ Department of Biosystems and Agricultural Engineering, Michigan State University, East Lansing, MI 48824-1323, USA. ${ }^{2}$ Department of Energy, Environmental and Chemical Engineering, Washington University in St. Louis, St. Louis, MO 63110, USA. ${ }^{3}$ Department of Biology, Heinrich Heine University, 40225 Düsseldorf, Germany.

\section{Acknowledgements}

The authors would like to thank Michigan AgBioResearch for funding this work through faculty salaries. The authors would also like to thank the Mass Spectrometry and Metabolomics Core of the Research Technology Support Facility at Michigan State University for their support on sample analysis.

\section{Competing interests}

The authors declare they have no competing interests.

\section{Availability of supporting data}

Data will be made available upon request.

\section{Funding}

This study is funded by the AgBioResearch at Michigan State University. WDH was supported by NSF (MCB 1616619).

Received: 10 September 2016 Accepted: 12 January 2017

Published online: 26 January 2017 


\section{References}

1. Bar-Even A, Noor E, Flamholz A, Milo R. Design and analysis of metabolic pathways supporting formatotrophic growth for electricity-dependent cultivation of microbes. Biochim Biophys Acta. 2013;1827(8-9):1039-47.

2. Kiehl JT, Trenberth KE. Earth's annual global mean energy budget. Bull Am Meteorol Soc. 1997;78(2):197-208.

3. Sundstrom ER, Criddle CS. Optimization of methanotrophic growth and production of poly(3-Hydroxybutyrate) in a high-throughput microbioreactor system. Appl Environ Microbiol. 2015;81(14):4767-73.

4. Trotsenko YA, Doronina NV, Khmelenina VN. Biotechnological potential of aerobic methylotrophic bacteria: a review of current state and future prospects. Appl Biochem Microbiol. 2005;41(5):433-41.

5. Rotatore C, Colman B. The active uptake of carbon dioxide by the unicellular green algae Chlorella saccharophila and C. ellipsoidea. Plant Cell Environ. 1991;14(4):371-5.

6. Jones JP, Prakash GK, Olah GA. Electrochemical $\mathrm{CO}_{2}$ reduction: recent advances and current trends. Isr J Chem. 2014;54(10):1451-66.

7. Li H, Opgenorth PH, Wernick DG, Rogers S, Wu TY, Higashide W, Malati P, Huo YX, Cho KM, Liao JC. Integrated electromicrobial conversion of $\mathrm{CO}_{2}$ to higher alcohols. Science. 2012;335(6076):1596.

8. Torella JP, Gagliardi CJ, Chen JS, Bediako DK, Colon B, Way JC, Silver PA, Nocera DG. Efficient solar-to-fuels production from a hybrid microbial-water-splitting catalyst system. Proc Natl Acad Sci USA. 2015;112(8):2337-42

9. Tremblay PL, Zhang T. Electrifying microbes for the production of chemicals. Front Microbiol. 2015;6:10.

10. Lidstrom ME, Stirling DI. Methylotrophs-genetics and commercial applications. Annu Rev Microbiol. 1990;44:27-58.

11. Bar-Even A, Noor E, Flamholz A, Milo R. Design and analysis of metabolic pathways supporting formatotrophic growth for electricitydependent cultivation of microbes. Biochim Biophys Acta Bioenerg. 2013;1827(8-9):1039-47.

12. Tai YS, Zhang KC. Enzyme Pathways $C 1$ metabolism redesigned. Nat Chem Biol. 2015;11(6):384-6.

13. Siegel JB, Smith AL, Poust S, Wargacki AJ, Bar-Even A, Louw C, Shen BW Eiben CB, Tran HM, Noor E, et al. Computational protein design enables a novel one-carbon assimilation pathway. Proc Natl Acad Sci USA. 2015;112(12):3704-9.

14. Atkinson PW, King JA, Hynes MJ. Identification of the ACIA gene controlled by the AMDA regulatory gene in Aspergillus nidulans. Curr Genet. 1985;10(2):133-8.

15. Babel W, Brinkmann U, Muller RH. The auxiliary substrate concept-an approach for overcoming limits of microbial performances. Acta Biotechnol. 1993;13(3):211-42.

16. Chow CM, Rajbhandary UL. Developmental regulation of the gene for formate dehydrogenase in Neurospora crassa. J Bacteriol. 1993;175(12):3703-9.

17. Bar-Even A. Formate assimilation: the metabolic architecture of natural and synthetic pathways. Biochemistry. 2016;55(28):3851-63.

18. Whitaker WB, Jones JA, Bennett RK, Gonzalez JE, Vernacchio VR, Collins SM, Palmer MA, Schmidt S, Antoniewicz MR, Koffas MA, et al. Engineering the biological conversion of methanol to specialty chemicals in Escherichia coli. Metab Eng. 2016:39:49-59.

19. Mueller JEN, Meyer F, Litsanov B, Kiefer P, Potthoff E, Heux S, Quax WJ, Wendisch VF, Brautaset T, Portais J-C, et al. Engineering Escherichia coli for methanol conversion. Metab Eng. 2015;28:190-201.

20. Lessmeier L, Pfeifenschneider J, Carnicer M, Heux S, Portais J-C, Wendisch VF. Production of carbon-13-labeled cadaverine by engineered Corynebacterium glutamicum using carbon-13-labeled methanol as co-substrate. Appl Microbiol Biotechnol. 2015;99(23):10163-76.

21. Bruinenberg PM, Jonker R, Vandijken JP, Scheffers WA. Utilization of formate as an additional energy-source by glucose-limited chemostat cultures of candida-utilis cbs-621 and Saccharomyces cerevisiae cbs8066 - evidence for the absence of transhydrogenase activity in yeasts. Arch Microbiol. 1985;142(3):302-6

22. Hazeu W, Donker RA. A continuous culture study of methanol and formate utilization by the yeast Pichia pastoris. Biotechnol Lett. 1983:5(6):399-404.

23. Babel W, Muller RH, Markuske KD. Improvement of growth-yield of yeast on glucose to the maximum by using an additional energy-source. Arch Microbiol. 1983;136(3):203-8.
24. Babel W. The auxiliary substrate concept: from simple considerations to heuristically valuable knowledge. Eng Life Sci. 2009;9(4):285-90.

25. Geertman JMA, van Dijken JP, Pronk JT. Engineering NADH metabolism in Saccharomyces cerevisiae: formate as an electron donor for glycerol production by anaerobic, glucose-limited chemostat cultures. FEMS Yeast Res. 2006;6(8):1193-203.

26. Harris DM, Van Der Krogt ZA, Van Gulik WM, Van Dijken JP, Pronk JT. Formate as an auxiliary substrate for glucose-limited cultivation of Penicillium chrysogenum impact on penicillin $\mathrm{G}$ production and biomass yield. Appl Environ Microbiol. 2007;73(15):5020-5.

27. Ruan Z, Zanotti M, Wang X, Ducey C, Liu Y. Evaluation of lipid accumulation from lignocellulosic sugars by Mortierella isabellina for biodiesel production. Bioresour Technol. 2012;110:198-205.

28. Bligh EG, Dyer WJ. A rapid method of total lipid extraction and purification. Can J Biochem Physiol. 1959;37(8):911-7.

29. Indarti E, Majid MIA, Hashim R, Chong A. Direct FAME synthesis for rapid total lipid analysis from fish oil and cod liver oil. J Food Compos Anal. 2005:18(2):161-70.

30. You L, Page L, Feng X, Berla B, Pakrasi HB, Tang YJ. Metabolic pathway confirmation and discovery through 13C-labeling of proteinogenic amino acids. J Vis Exp. 2012;59:e3583.

31. King ZA, Lu J, Drager A, Miller P, Federowicz S, Lerman JA, Ebrahim A, Palsson BO, Lewis NE. BiGG models: a platform for integrating, standardizing and sharing genome-scale models. Nucleic Acids Res. 2016:44(D1):D515-22

32. Dauner M, Sauer U. GC-MS analysis of amino acids rapidly provides rich information for isotopomer balancing. Biotechnol Prog. 2000;16:642-9.

33. Wu G, Yan Q, Jones JA, Tang YJ, Fong SS, Koffas MA. Metabolic burden: cornerstones in synthetic biology and metabolic engineering applications. Trends Biotechnol. 2016;34:652-64.

34. Brosnan ME, MacMillan L, Stevens JR, Brosnan JT. Division of labour: how does folate metabolism partition between one-carbon metabolism and amino acid oxidation? Biochem J. 2015:472(2):135-46.

35. Feisthauer S, Wick LY, Kastner M, Kaschabek SR, Schlomann M, Richnow $\mathrm{HH}$. Differences of heterotrophic $\left(\mathrm{CO}_{2}\right)-\mathrm{C}-13$ assimilation by Pseudomonas knackmussii strain B13 and Rhodococcus opacus 1CP and potential impact on biomarker stable isotope probing. Environ Microbiol. 2008;10(6):1641-51.

36. Dijkstra AJ, Hamilton RJ, Hamm W. Trans fatty acids. New York: Wiley; 2008

37. Froissard $M$, Canonge $M$, Pouteaux $M$, Cintrat B, Mohand-Oumoussa S, Guillouet SE, Chardot T, Jacques N, Casaregola S. Lipids containing medium-chain fatty acids are specific to post-whole genome duplication Saccharomycotina yeasts. BMC Evol Biol. 2015;15:1.

\section{Submit your next manuscript to BioMed Central and we will help you at every step:}

- We accept pre-submission inquiries

- Our selector tool helps you to find the most relevant journal

- We provide round the clock customer support

- Convenient online submission

- Thorough peer review

- Inclusion in PubMed and all major indexing services

- Maximum visibility for your research

Submit your manuscript at www.biomedcentral.com/submit
C BioMed Central 